\title{
Frecuencia y tipificación de anticuerpos irregulares en un servicio de medicina transfusional nivel IV, Cali
}

- Henry Idrobo Quintero, Nora Bolívar Cuten, Juan Manuel Herrera, Álvaro Jaime Guerrero Villota, Érika Rincón, Camilo Ernesto Zorrilla, Lina Zapata, Érika Cantor, Alexander Martínez, residentes de hematooncología de la Universidad Libre, docentes de hematooncología de la Universidad Libre

Centro Médico Imbanaco, Cali (Valle del Cauca).

Contacto: henryidroboquintero@gmail.com

Introducción y objetivos. La identificación de anticuerpos irregulares $(\mathrm{ACl})$ es importante para evitar reacciones hemolíticas. Objetivo: identificar Acl hallados durante pruebas pretransfusionales en una institución de alta complejidad de Cali en un período de seis años.

Materiales y métodos. Estudio retrospectivo de corte transversal. Se revisaron 15.781 registros de inmunohematología pretransfusional durante 2007-2013, encontrándose 240 casos con Acl en población no gestante. Se comparó la proporción de Acl por género, grupos etarios, diagnósticos, grupo sanguíneo.

Resultados. La prevalencia de Acl fue del $1 \%$ en 2008 y del $2 \%$ en 2013 , identificándose a una edad promedio de 50 años (DE \pm 26 ), el $58,7 \%$ entre $51-94$ años y el $72,5 \%$ en mujeres. Los grupos sanguíneos más frecuentes fueron $\mathrm{O}(59 \%)$ y $\mathrm{A}(27,2 \%), 81,2 \% \mathrm{Rh}(+)$. El
$23,3 \%$ presentó autoanticuerpos y el $85,8 \%$, aloanticuerpos (AloAcl). En el 15,5\% se tipificó más de un Alo-Acl. Los Acl más frecuentes fueron anti-E $(17,5 \%)$, anti-K (14,2\%) y anti-D (12,9\%). Anti-cC-M-Fya-e-Lea-Jka-Jkb variaron entre el $2 \%$ y $6 \%$. En el $6,3 \%$ no se identificó el tipo de Acl. Para los tres anticuerpos más frecuentes, no hubo diferencias significativas respecto a género y grupo sanguíneo, pero fue más frecuente anti- $K$ en pacientes sometidos a cirugías y mayores de 50 años, y anti-D en pacientes con anemias no hemolíticas y edades de 10-50 años.

Conclusiones. Aumento de la prevalencia de Acl en el tiempo. El número de Alo-Acl, género, edad y Acl más frecuentes se comportaron similar a lo reportado, pero en otras variables hubo diferencia, lo que sugiere necesidad de validar técnicas en nuestra población. El grupo etario y el diagnóstico pueden influir en el tipo de Acl.

\section{Construcción de la matriz de atención multiprofesional del paciente pre y pos-TCMH y sus repercusiones en la asistencia}

- Cristiane Olmos Grings, Ana Maria Keller Jochims, Joice Zuckermann, Camila Oppermann, Débora Moraes, Rita Maria Soares, Regina Sikilero, Carlos Orozco Oviedo, Alessandra Paz, Lucia da Rocha Silla

Hospital de Clínicas de Porto Alegre, Brasil.

Contacto: carlosorozco73@hotmail.com

Introducción y objetivos. Por ser un tratamiento de alta complejidad, el trasplante de células madres hematopoyéticas (TCMH) requiere de la actuación integrada del equipo asistencial. Por lo tanto, el objetivo de este estudio es describir la matriz multiprofesional y el impacto sobre el proceso de atención del equipo involucrado en el cuidado de los pacientes de TCMH.

Materiales y métodos. A partir de las preguntas sobre la evaluación pre-TCMH, se estructuró un grupo de trabajo integrado por médicos, enfermeras, nutricionista, farmacéutico clínico, psicólogo, fisioterapeuta, trabajo social, maestro de educación física, odontólogo, profesional de bioética y administrador, con el objetivo de discutir el flujo de pacientes y las diferentes funciones e interrelaciones de los profesionales que intervienen en las etapas del proceso. Fue elaborada la matriz de atención mulitiprofesional del paciente pre y pos-TCMH, en la que se describen las actividades y atribuciones de cada profesional, la cual contribuyó para la reestructuración de la asistencia.

Resultados. Con base en la identificación de los aspectos peculiares y compartidos de cada etapa del proceso descrito en la matriz, en el año 2013, fue oficializado el Programa Asistencial de Trasplante de Células Tronco Hematopoyéticas (PATCTH) para promover, organizar y perfeccionar acciones multidisciplinarias relacionados con $\mathrm{TCMH}$, teniendo en cuenta la complejidad del proceso y las características específicas de las profesiones en la atención integral y humana del paciente.

Conclusiones. Dada la complejidad de la asistencia de los pacientes sometidos a TCMH, es necesaria la conformación de equipos multiprofesionales e interdisciplinarios para ofrecer una atención integral. 